\title{
Absolute Measurement of Elastic Waves Excited by Hertzian Contacts in Boundary Restricted Systems
}

\author{
S. Schnabel $1^{1} \cdot$ S. Golling ${ }^{2} \cdot$ P. Marklund ${ }^{3} \cdot$ R. Larsson $^{3}$
}

Received: 9 August 2016/Accepted: 17 November 2016/Published online: 25 November 2016

(c) The Author(s) 2016. This article is published with open access at Springerlink.com

\begin{abstract}
In most applied monitoring investigations using acoustic emission, measurements are carried out relatively, even though that limits the use of the extracted information. The authors believe acoustic emission monitoring can be improved by instead using absolute measurements. However, knowledge about absolute measurement in boundary restricted systems is limited. This article evaluates a method for absolute calibration of acoustic emission transducers and evaluates its performance in a boundary restricted system. Absolute measured signals of Hertzian contact excited elastic waves in boundary restricted systems were studied with respect to contact time and excitation energy. Good agreement is shown between measured and calculated signals. For contact times short enough to avoid interaction between elastic waves and initiating forces, the signals contain both resonances and zero frequencies, whereas for longer contact times the signals exclusively contained resonances. For both cases, a
\end{abstract}

S. Schnabel

stephan.schnabel@1tu.se

1 Division of machine elements, SKF-University technology center, Luleå University of Technology, 97187 Luleå, Sweden

2 Division of solid mechanics, Luleå University of Technology, 97187 Luleå, Sweden

3 Division of machine elements, Luleå University of Technology, 97187 Luleå, Sweden
Green's function model and measured signals showed good agreement.

Keywords Hertz contact - Elastic waves - Acoustic emission · Green's function - Boundary restricted system · Condition monitoring

\section{List of Symbols}

$c_{\mathrm{p}} \quad$ Speed of elastic pressure wave

$E_{1}, E_{2} \quad$ Elastic modulus ball (1), plate disc (2)

$f_{\text {amp }} \quad$ Max force measured during calibration test

$f_{j} \quad$ Force in direction $j$

$f_{\max } \quad$ Maximum Hertzian force

$f_{\text {norm }, N} \quad$ Normalized zero frequencies of $N$-oder

$f_{\text {zero }, N} \quad$ Zero frequencies of $N$-oder

$g_{k j} \quad$ Green's function from direction $j-k$

$h \quad$ Thickness/height of plate/disc

$i_{1} \quad$ Sensor function time domain in direction 1

$I_{1} \quad$ Sensor function frequency domain in direction 2

$R \quad$ Ball radius

$r_{\text {disc } \quad \text { Disc radius }}$

$s \quad$ Measured raw signal, time domain

$S \quad$ Measured raw signal, frequency domain

$t \quad$ Time (measurement base)

$t_{c} \quad$ Hertzian contact time

$t_{\text {reflection } H}$ Reflection time across plate/disc height

$t_{\text {reflection } R}$ Reflection time across disc radius

$t_{\text {rise }} \quad$ Rise time of step function

$u_{k} \quad$ Displacement in time domain in direction $k$

$U_{k} \quad$ Displacement in frequency domain in direction $k$

$v_{0} \quad$ Impact velocity

$x \quad$ Position of sensor

$\delta_{1}, \delta_{2} \quad$ Material factor for ball (1) and plate/disc (2)

$v_{1}, v_{2} \quad$ Poisson's ratio ball (1), plate/disc (2) 


$\begin{array}{ll}\xi & \text { Position of impact } \\ \rho_{1}, \rho_{2} & \text { Density ball (1), plate/disc (2) } \\ \tau & \text { Time (impact base) } \\ \omega & \text { Frequency }\end{array}$

\section{Introduction}

Acoustic emissions (AE), or as well called high frequency elastic wave emissions, have over the past decade become increasingly popular in the application of nondestructive testing and condition monitoring. This technique has been proven in the separation of failure modes [2], the monitoring of wear [4, 21], the specification of contaminated systems [16, 20] and to differentiate lubricants [17] and lubrication regimes [7]. However, most of the investigations use simple signal processing methods such as root mean square (RMS) [7, 20] and activation counts (AC) $[2,16,17,21]$. All these investigations use relative measurement methods, and signals are acquired using piezoelectric transducers, which limits investigations without further calibration to measure relatively. This use of relative measurement methods also limits the extraction of information of the acoustic wave.

The authors hypothesize that condition monitoring techniques could be improved by improving processing of the signal. A fuller understanding of the relation between the source of the wave and the actual measured signal would improve the processing of the signal. Being able to calculate the force function of the initial source based on sensor signals would increase the possibility to distinguish between different failure types and failure sizes. However, absolute measurement would therefore be required so that the relation between the signal and the wave source could be ascertained. Both McLaskey and Glaser [15] and Jacobs and Woolsey [10] have presented methods for absolute calibration of piezoelectric transducers. However, there are no evaluations of the validity of the methods which are independent of the system. McLaskey's and Glaser's method for absolute calibration of piezoelectric transducers is used. The method is evaluated for boundary restricted systems using a Laser-Doppler vibrometer (LDV) and Green's function based on an FEM simulation as a comparison. The term "boundary restricted systems" is used for systems where reflections of all dimensions are taken into account (in this investigation disc samples), whereas systems which are boundary free in one or two dimension are not included in this definition (calibration plate-reflection is only considered in one dimension).

An absolute measurement is required to improve condition monitoring capability of high frequency emissions. However, a better understanding of the relation between source and signal is as well an essential knowledge for improving condition monitoring by acoustic emission. Several researchers have successfully connected source and signal for high frequency emissions. McLaskey and Glaser [14] have, for example, related a signal of a piezoelectric transducer to the actual force function by using a Green's function approach. Kundu et al. [12] have presented a mathematical method to locate Hertzian impacts by minimizing error functions. The impact of cracks on wave propagation in plates was studied by Liu and Datta [13] with FEM based on a Green's function for transfer of the initial source. Glaser et al. [5] were able to calculate the wave propagation in an isotropic half space with a viscoelastic propagator and compared it to actual measurements. All these investigations either have used thin plates in order to minimize the problem to two dimensions or have used huge geometries in comparison with the measurement time to avoid reflections. In both cases, the boundary restrictions are simplified. However, in industrial applications boundary restrictions often comprise a great part of contribution to the measured signal.

Other researchers have taken boundary restrictions into account by using smaller geometries with limited sizes, where reflection could not be neglected, but have focused on linking physical properties to the measured signal rather than the actual source. Han et al. [8], for example, related the yield stress in tensile tests with wave forms, while Niknam et al. [18] related AE signals with a statistical approach to lubrication conditions. However, in these investigations absolute measurement is not used nor do they relate signal and source directly to each other.

Investigations with boundary restricted systems which relate wave source and signal are rare. One of the few that do is an investigation about crack propagation in brittle amorphous materials, where Gross et al. [6] link the spectra of the excitation to the speed of the crack propagation. However, investigations of Hertzian contact excited, boundary restricted systems, could not be found, even though such systems are fundamental for condition monitoring of rolling element bearings. Understanding the relation between $\mathrm{AE}$ source and $\mathrm{AE}$ signals in a rolling element bearing would enable new opportunities for improved signal processing. This article therefore describes a experimental analysis of Hertzian contacts in boundary restricted systems and compares the results to previous investigations without boundary restrictions.

\section{Experimental Setup}

Steel balls (SKF) with diameters from $\varnothing 1.5$ to $\varnothing 10 \mathrm{~mm}$ were used as Hertzian excitation for all experiments. The balls were dropped from different heights onto different plates (Table 1). Impact positions were identical for all 
Table 1 Test setup

\begin{tabular}{lll}
\hline Disc samples & Ball samples & Drop heights (mm) \\
\hline Aluminium $70755 \mathrm{~mm}$ & RB1.5 G20 & $100 \mathrm{~mm}$ \\
Aluminium $70757 \mathrm{~mm}$ & RB2 G20 & $150 \mathrm{~mm}$ \\
Steel $25115 \mathrm{~mm}$ & RB3 G20 & $250 \mathrm{~mm}$ \\
Steel $25117 \mathrm{~mm}$ & RB4 G20 & $350 \mathrm{~mm}$ \\
Steel $251129 \mathrm{~mm}$ & RB6 G20 & \\
& RB8 G20 & \\
& RB10 G20 \\
\hline
\end{tabular}

experiments so as to obtain repeatable measurements. To achieve as identical impact positions as possible, a hemispherical magnetic holder was developed (Fig. 1). With a magnetic force just enough to overcome gravity, the ball samples were self-aligning by positioning at lowest potential energy, right centred beneath the half sphere. Any redirection problem due to residual magnetism was minimized due to the minimization of contact area. This repeatability is visualized in Fig. 2. It shows repeated drops onto a pressure sensitive paper from various heights. The spread of contact points increases with increasing drop height. However, the results demonstrate an acceptable repeatability, especially considering that the ball penetrates the 0.1-mm-thick pressure sensitive paper.

Another adjustment of the test setup was the position of sensors (LDV and AE transducer, respectively) relative to the position of the impact. Using a Green's function approach, it is important to have impact point and sensor point vertically aligned to each other (according to Fig. 3, points $\xi$ and $x$ ). To achieve this setup, the laser was aligned vertically by aligning the reflection of a water bath. If the laser was reflected back to its source by the water surface, the laser hit the water surface at a $90^{\circ}$ angle and was thereby vertically aligned. Once the laser was vertically aligned, the magnetic holder was centred to the laser. Therefore was the effect used that the reflection right back

(a)
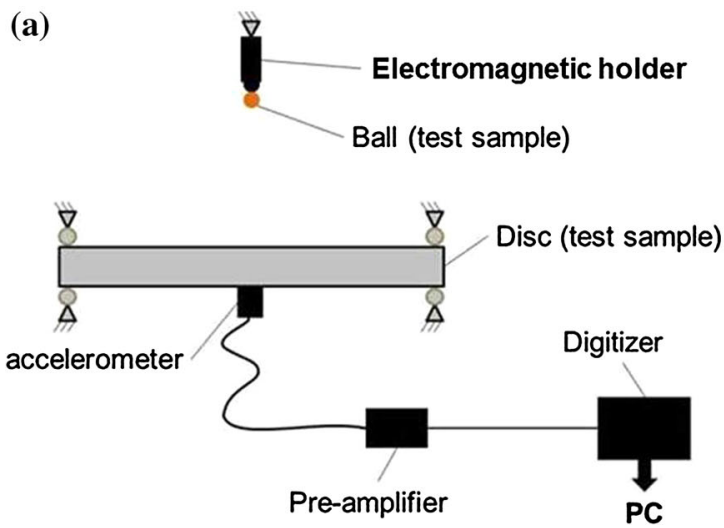

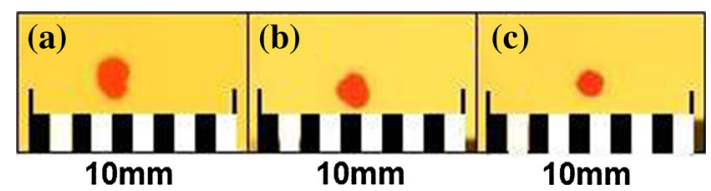

Fig. 2 Drop tests for evaluation of repeatability with pressure sensitive paper. a $350 \mathrm{~mm}$ drop height, b $250 \mathrm{~mm}$ drop height, c $150 \mathrm{~mm}$ drop height

to the laser source is maximized, when the laser hit the centre point of the sphere.

As shown in Fig. 1, the various samples (discs) are clamped between two rubber o-ring seals. In this investigation, five different discs were used and excited by different Hertzian impacts as shown in Table 1. The discs samples only differ in material and thickness. Both diameter $(\varnothing 103.8 \mathrm{~mm})$ and a $30^{\circ}$ chamfer on one side were identical for all samples. Several combinations of disc and ball samples were tested and measured with both the Laser-Doppler vibrometer and the piezoelectric AE transducer. Each measurement was repeated eight times. All samples and test equipment, except pre-amplifier and acquisition computer, were mounted on an optical table to minimize disturbances of the surrounding.

$\mathrm{AE}$ transducer measurements were executed with the same broadband flat-response sensor (Physical acoustics $\mathrm{WS} \alpha$ ) used with the calibration measurements. As a preamplifier, a Physical Acoustic 2/4/6C was used with an

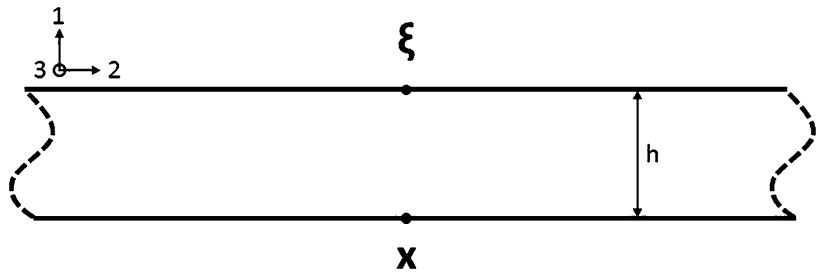

Fig. 3 Schematic for declaration of experimental setup

(b)
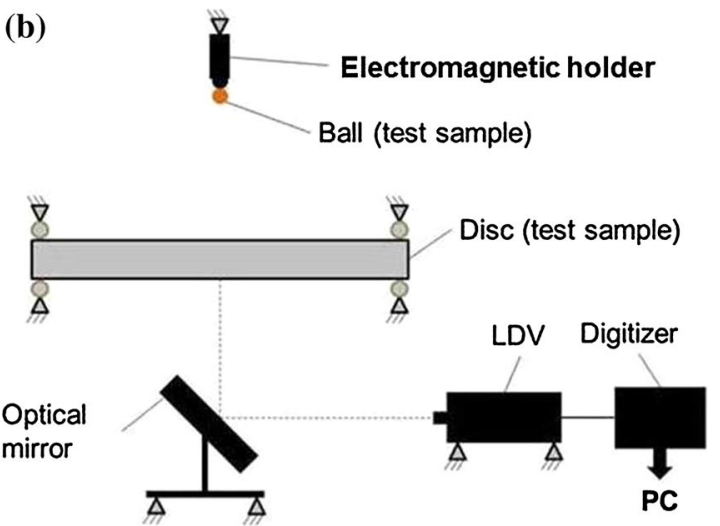

Fig. 1 Schematic drawings of the experimental setups. a AE transducer setup. b LDV setup 
inbuilt bandpass filter of $100-1200 \mathrm{kHz}$. The sensor was attached using beeswax. Therefore, beeswax was both the attachment and the couplant. A small portion was heated to $75^{\circ} \mathrm{C}$. Then the sensor was pressed with dead weights onto the discs, while waiting until both disc and couplant reached room temperature again. Beeswax was chosen following tests with various methods for the attachments prior to the investigation. Additionally, several other researchers have in previous tests used beeswax as a couplant for acoustic measurements $[3,11]$.

The laser measurement was executed with a device called OFV056 from Polytec. The OFV056 is a laboratory type Laser-Doppler vibrometer (LDV) which uses a red laser. The LDV measures the velocity of a single point on the surface of the discs (Fig. 1a), which was by the provided software converted into displacement. In this investigation, a sensitivity of $(125 \mathrm{~mm} / \mathrm{s}) / \mathrm{mV}$ was used in combination with a measurement range of $1 \mathrm{~Hz}-1 \mathrm{MHz}$. The laser head was used in combination with a matching controller and pre-amplifier box (Polytec OFV3001S). As shown in Fig. 1, it was necessary to redirect the laser by an optical mirror (Thorlabs PF20-03-P01).

The signals of both the LDV and the AE transducer are stored with a 14-bit digitizer PCI-card (GaGe OSC-432007). A sample rate of $10 \mathrm{MS} / \mathrm{s}$ was used and in total 10000 samples per signal were recorded, which resulted in a measurement time of $1 \mathrm{~ms}$. However, limited to a measurement time of $100 \mu \mathrm{s}$, all signals were triggered on a positive flank with a pretrigger storage of $1 \mu \mathrm{s}$.

\section{Green's Function Approach}

Both the calibration and simulation are based on a Green's function approach, where a single function describes the time-dependent relation between two physical measures at two points within the system boundaries. Usually, the Green's function $g$ is a matrix which describes the relationship between points $(x, \xi)$ for given directions $(k, j)$ at specific times $(t, \tau)$ :

$u_{k}(x, t)=\iint f_{j}(\xi, \tau) \times g_{k j}(x, t, \xi, \tau) \mathrm{d} \xi \mathrm{d} \tau$

However, in this paper a simplified case is used, such as that which McLaskey and Glaser suggest [14]. It is assumed that the major contribution is the pressure wave through the material and therefore the simplified Green's function is only dependent on a single direction. As shown in Fig. 3, the two points of interest are only separated in direction 1 by the distance $h$. For all other directions, the distance between $x$ and $\xi$ is zero. All exciting forces $f$ are assumed to be perpendicular to the plate, and forces therefore only act along direction 1 . The same applies for the displacement $u$. If these assumptions are made, Green's function may be simplified according to Eq. (2) as shown by McLaskey and Glaser [14]:

$u_{1}(x, t)=f_{1}(\xi, \tau) * g_{11}(x, t, \xi, \tau)$

Equation (2) allows determination of the displacement $\left(u_{1}(x, t)\right)$ in the perpendicular direction to the plate at the sensor position, by convolution of Green's function $\left(g_{11}(x, t, \xi, \tau)\right)$ and given force functions $\left(f_{1}(\xi, \tau)\right)$. The Green's function approach presented in this report requires independence between force function and Green's function. Therefore, calculations are only valid for contact times $\left(t_{\mathrm{c}}\right)$ shorter than any reflection time back to the point of force initiation $(\xi)$. This is especially important for the boundary restricted systems (in this investigation small discs) and reflection times are therefore defined as follows:

$$
\begin{aligned}
& t_{\text {reflection } H}=2 \frac{h}{c_{\mathrm{p}}} \\
& t_{\text {reflection } R}=2 \frac{r_{\text {disc }}}{c_{\mathrm{p}}}
\end{aligned}
$$

where $t_{\text {reflection } H}$ and $t_{\text {reflection } R}$ are reflection times across the disc and radial, respectively. The variables $h$ and $r_{\text {disc }}$ are height and radius of the disc, and $c_{\mathrm{p}}$ is the speed of the pressure wave in the given material.

The Green's function approach as used in this investigation is based on two point assumptions: One assumption is that the global force of a Hertzian elastic contact model as shown in Eq. (8) acts on a single point (in this investigation $\xi$ ). This assumption requires a small contact area and a deformation that is as homogeneous as possible across the contact area. The second point assumption used in this investigation is that calculated displacements of a single point (in this investigation $x$ ) represent measured acoustic waves by a sensor with a finite contact area. This assumption requires a ratio between sensor diameter and distance of the measurement point $(x)$ to source point $(\xi)$ as small as possible.

\section{Calibration}

As seen in Fig. 4, even high quality AE transducers with flat-response characteristic are not perfectly flat. These small variations within the bandwidth of the sensor, in case of the WS $\alpha 0.1-1 \mathrm{MHz}$, do have an influence once the sensor is used for absolute measurement instead of relative measurement. Additionally, the amplitude needs to be related to a physical measure once absolute measurements are carried out. Therefore, a calibration is necessary once the sensor is used for absolute measurements and once signals will be compared to simulations. 


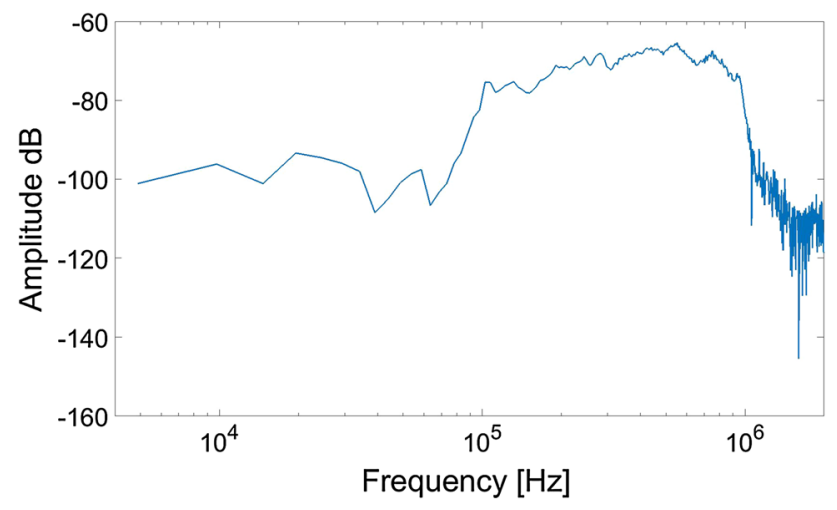

Fig. 4 Frequency response function of the AE transducer provided by the supplier

McLaskey's and Glaser's [15] method was used for calibration of the AE transducer. As a calibration system a steel plate (Toolox40) with dimensions of $1 \times 0.5 \times, 0.051 \mathrm{~m}$ was used. The huge dimensions of the steel plate were necessary in order to avoid reflections from the side walls during the measurement time of $80 \mu \mathrm{s}$. Being able to neglect reflections allowed the use of Hsu's [9] script for calculating Green's function, which uses only material properties and the thickness of the plate as input parameters. The source for the wave was a glass capillary tube $(D=\varnothing 1.4 \mathrm{~mm}$ and $d=\varnothing 1 \mathrm{~mm}$ ) which was compressed until it burst on the surface of the plate. A glass capillary burst was chosen as a source because of its good excitation of high frequencies due to the rapid release of force [1] and that the following equation may be used:

$f(\tau)=\frac{f_{\mathrm{amp}}}{2 \times\left(1-\cos \left(\pi \tau / t_{\text {rise }}\right)\right)}$,

$f(\tau)=0$,

$f(\tau)=f_{\mathrm{amp}}$

The calibration was carried out in a tensile test machine, where the steel plate was positioned under a steel cylinder and pressed against it with the glass capillary in between. With a speed of $0.01 \mathrm{~mm} / \mathrm{s}$, the plate was elevated towards the steel cylinder and the force on the glass capillary as a result increased successively. During the whole calibration, the force on the steel cylinder, and thereby the force on the glass capillary, was measured continuously with a sample rate of 50 Samples/s. With the force measurement, the force, at which the glass capillary burst, was identified by the force maximum. This force maximum was the input value for $f_{\text {amp }}$ of Eq. (5). The released elastic wave was measured with an AE transducer (Physical acoustics WS $\alpha$ ), which was positioned centred to the source underneath the steel plate (positions comparable to $\mathrm{x}$ in Fig. 3). By transforming the measured AE transducer signal $s(x, t)$ and the calculated theoretical displacement $u_{1}(x, t)$ to the frequency plane, the sensor function is calculated by simple division [15]:

$$
\begin{aligned}
& s(x, t)=u_{1}(x, t) * i_{1}(t) \\
& I_{1}(\omega)=S(x, \omega) / U_{1}(x, \omega)
\end{aligned}
$$

While in the time domain the sensor signal $(s(x, t))$ is the result of the convolution of the actual displacement $\left(u_{1}(x, t)\right)$ and the sensor function $\left(i_{1}(t)\right)$, the convolution will transform into simple multiplication. Therefore, the sensor function $\left(I_{1}(\omega)\right)$ in the frequency domain can be extracted by dividing sensor signal $(S(x, \omega))$ and displacement $\left(U_{1}(x, \omega)\right)$.

The sensor function is the function transforming displacement of the surface to the sensor signal, including both sensor and amplifier. This transfer function can be called as well frequency response function. However, due to the definition of the used calibration method [15] it is called sensor function throughout the article. Directions of force and displacement as well as relative positions of source and measurement point were kept constant for all experiments. The extracted sensor function was therefore valid for all measured signals with the AE transducer. Further signals compensated with the sensor function represent spectra of the displacement of the point of measurement. Therefore, compensated spectra represent absolute values and enable comparison with spectra of simulated signals. This calibration method does not only allow absolute measurement, it also influence the definition of AE. In this article, AE is defined as a movement over time of a measurement point with a frequency higher than $20 \mathrm{kHz}$, regardless source.

\section{Simulation}

A simulation was carried out to be compared with experimental findings. Therefore, the spectra of the displacement $U_{1}(x, \omega)$ were studied. First $u_{1}(x, t)$ was calculated by convolution of $f_{1}(\xi, \tau)$ and $g_{11}(x, t, \xi, \tau)($ Eq. 2$)$. For the force function, an equation based on Hertz elastic contact mechanics derived by Reed [19] was used:

$$
\begin{array}{ll}
f(\tau)=f_{\max } \sin \left(\pi \tau / t_{c}\right)^{3 / 2}, & 0 \leq \tau \leq t_{c} \\
f(\tau)=0, & \tau<0 \text { and } \tau>t_{c}
\end{array}
$$

Maximum force $\left(f_{\max }\right)$ and contact time $\left(t_{c}\right)$ are calculated based on geometrical and physical properties according to the following equations:

$$
f_{\max }=1.917 \rho_{1}^{3 / 5}\left(\delta_{1}+\delta_{2}\right)^{-2 / 5} R_{1}^{2} v_{0}^{6 / 5}
$$




$$
\begin{aligned}
t_{c} & =4.53\left(4 \rho_{1} \pi\left(\delta_{1}+\delta_{2}\right) / 3\right)^{2 / 5} R_{1} v_{0}^{-1 / 5} \\
\text { where } \delta_{i} & =\frac{1-v_{i}^{2}}{\pi E_{i}}
\end{aligned}
$$

The indexes in Eqs. (9) and (10) refer to the ball (index 1) and the disc (index 2), while index 0 refers to the velocity at the time 0 (initial impact velocity $v_{0}$ ).

Knowing materials and geometrical properties of the samples allows the calculation of an analytical solution for the force function. However, a solution for Green's function $\left(g_{11}(x, t, \xi, \tau)\right)$ of the used samples where reflections could not be neglected was neither solvable analytically nor by the Hsus [9] script used for calibration. Therefore, a finite element method (FEM) analysis was carried out to generate Green's functions for disc samples used in this investigation. The FEM analysis was based on an LS DYNA solver for rotational symmetry. The element size of the rectangular elements was $50 \mu \mathrm{m}$. Time steps are usually connected to element size and material properties in LS DYNA, which results in $3.6 \mathrm{~ns}$ for steel and $3.7 \mathrm{~ns}$ for aluminium. However, in this investigation a storage interval of $10 \mathrm{~ns}$ was used to artificially increase the time step and thereby to homogenize the time step for all samples.

Figure 5 shows a schematic drawing of the FEM model with the red symmetry axis on the left side. The three yellow marked nodes on the top (marked with F) of the disc in Fig. 5 represent the nodes for the force initiation. The nodes were exposed to a step force with a rise time of $100 \mathrm{~ns}$. The three nodes form a circular area with a radius of $100 \mu \mathrm{m}$, where the maximum force applied is $1 \mathrm{~N}$ over this circular area. Usually Green's function approaches are transfer functions between two points. However, to obtain convergence, the FEM model uses three points for the force initiation. The stored output of the model is the displacement in $y$-direction of a single node on the lower side of the disc positioned on the symmetry axis (Yellow node marked with $M$, Fig. 5). A boundary constrain was introduced to take the fixation of the discs into account. A single node with a distance to the symmetry axis equivalent to the

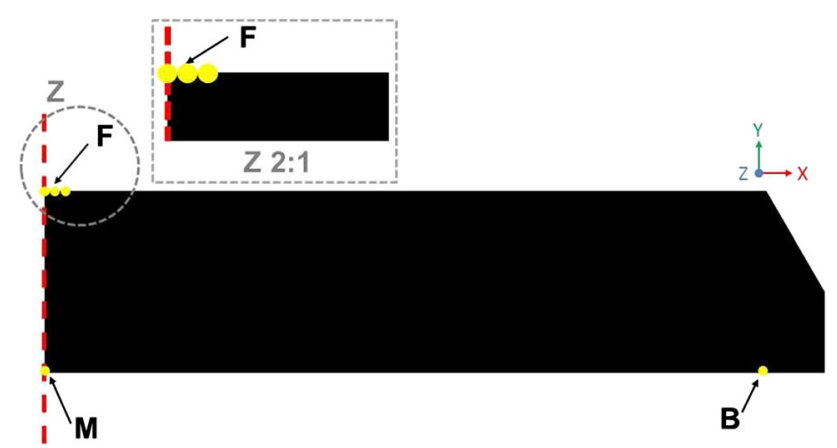

Fig. 5 Schematic drawing of the FEM model with the symmetry axis and nodes for loading $(F)$, response extraction $(M)$ and constraint $(B)$ radius of the rubber seals was tied in the $y$ - and the $z$ direction (Yellow node marked with $B$, Fig. 5). By taking the derivative of the FEM-result (displacement in $y$-direction of node $M$ ), Green's function for specific samples was obtained. In combination with the equation for the force function (8), it enabled the calculation of the spectra for various Hertzian impacts. However, simulated results were only valid as long as the force and elastic wave do not $\operatorname{interact}\left(t_{c}<t_{\text {reflection } H}, t_{\text {reflection } R}\right)$.

\section{Zero Frequencies}

Zero frequencies according to McLaskey and Glaser [14] are calculated by using Eq. (11). McLaskey and Glaser [14] have shown acoustic waves excited by a Hertzian contact have local minima in the signal spectra (Fig. 6$\left.f_{\text {zero, } 1}, f_{\text {zero }, 2}, f_{\text {zero }, 3}\right)$ which they call zero frequencies. These zero frequencies are determined by the contact time $\left(t_{c}\right)$ and have different $\operatorname{orders}(N=[1,2,3,4, \ldots])$ which are in theory infinite.

$$
f_{\text {zero }, N}=\frac{N+0.75}{t_{\mathrm{c}}}
$$

However, in practice measurement of zero frequencies at higher orders is difficult due to higher damping of higher frequencies. This study therefore also focuses, as do McLaskey and Glaser [14], on zero frequencies up to the third order.

\section{Results and Discussion}

Results are divided in two sections. After repeating earlier results [14] to confirm that the calibration, by McLaskey's and Glaser's [15] method, was correctly executed, the calibration method was evaluated for boundary restricted systems. Therefore, measured spectra of acoustic waves in

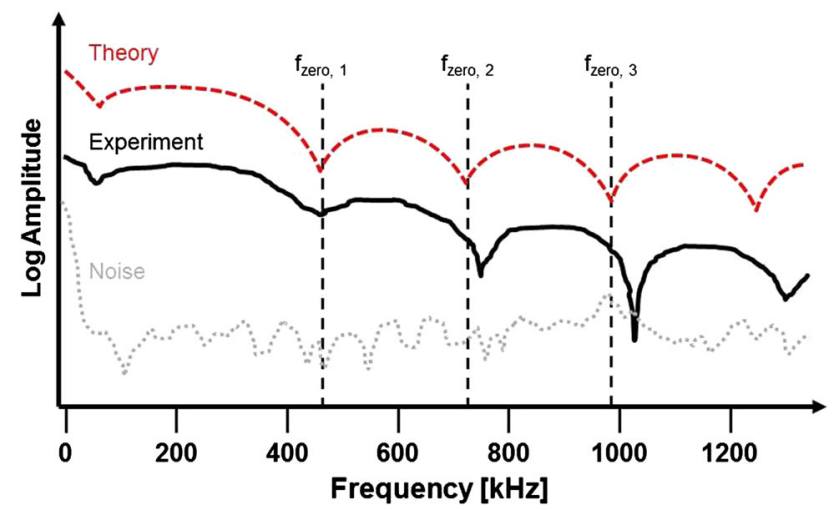

Fig. 6 Schematic replication of results obtained by McLaskey and Glaser [14] 
disc samples were compared to FEM simulated spectra and LDV measured spectra. In the second section, results on the behaviour of Hertzian excited elastic waves in boundary restricted systems will be shown. For cases where source and wave interact with each other, LDV measured spectra are presented and analysed. In the case of independent source and wave, AE transducer measured spectra are shown, analysed and compared to FEM simulations with respect to zero frequencies.

\subsection{Evaluation of Absolute Calibration for Boundary Restricted Systems}

As mentioned in Sect. 4, a calibration method by McLaskey and Glaser [15] was used to extract the sensor function $I_{1}(\omega)$. To confirm that the calibration was executed correctly, McLaskey's experiment with Hertzian contacts was repeated. Figure 6 shows the results to be repeated, and Fig. 7 shows the repeated experiment for control of the calibration. The theoretical signal (green) is calculated with Hsus script and Eqs. (2)-(10). In Fig. 7a, a clear difference between the measured raw signal and the calculated theoretical signal can be seen. However, after compensating for the sensor function $I_{1}(\omega)(7)$, the measured AE transducer signal (red) and the theoretical signal (green) show a good agreement (Fig. 7b) between $20 \mathrm{kHz}$ and $1.2 \mathrm{MHz}$. Even the zero frequencies $\left(f_{\text {zero, } n}\right)$ of the theoretical signal and the measured AE transducer signal match each other. Comparing to McLaskey's and Glaser's experiments, the zero frequencies are positioned at similar positions considering plate material and ball sample. Both the overall agreement and the agreement of the zero frequencies (Fig. 7b) indicate a successful calibration and extraction of the sensor function and are in line with the earlier result of McLaskey and Glaser (Fig. 6). The difference in zero frequencies between the two results is caused by different plate materials (aluminium vs. steel) and ball sizes (1 vs. $1.5 \mathrm{~mm}$ ) which results in different contact times $t_{c}$. Therefore, the

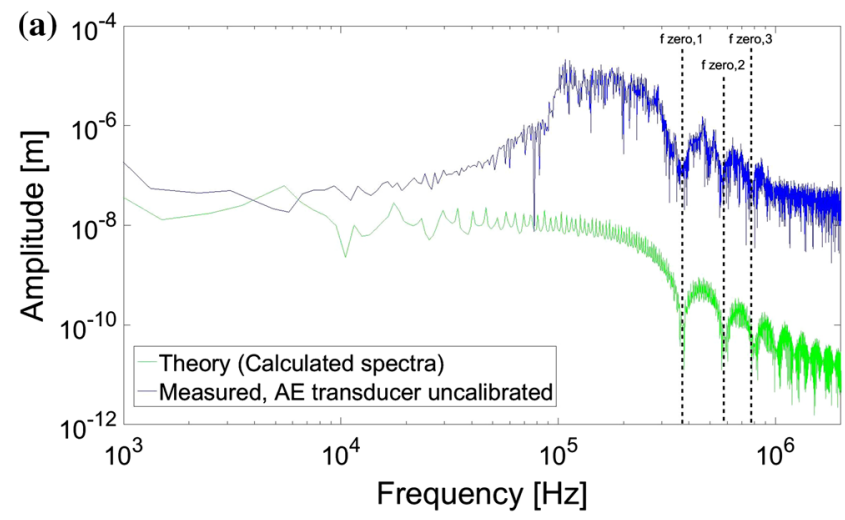

Fig. 7 Calibration evaluation a raw signal, b calibrated signal difference is expected and does not affect the conclusion of a successful calibration.

The extracted and controlled sensor function was then used for compensation of AE transducer signals recorded from Hertzian excitations in boundary restricted systems. These compensated signals were then compared to FEM simulation and LDV measured signals. For cases where source and wave do not interact with each other, the FEM simulated signal was used as a reference, while LDV measured signals were the reference for cases where source and wave do interact. The use of two different references was necessary, because for thick discs the displacement was too small to be detected by the LDV and the interaction of wave and source in thin discs could not be managed by the FEM simulation. Figure 8a shows both the LDV measured and compensated AE transducer measured signal of a Hertzian impact ( $\varnothing 8 \mathrm{~mm}$ Ball) on a 5 -mm-thick aluminium disc. Geometrical dimensions lead to multiple reflections ( $t_{\text {reflection } H}=1.575 \mu \mathrm{s}$ and $t_{\text {reflection } R}=16.346 \mu \mathrm{s}$ ) within the disc in all directions during the contact time of $31.96 \mu \mathrm{s}$. Therefore, excitation force and resulting wave are interacting with each other. As a result, the Hertzian impact excites resonances of the plate rather than enhancing zero frequencies. For this case, the calibration method shows weaknesses (Fig. 8a). While the LDV measured signal (purple) clearly shows the excitation of the resonances, the compensated AE transducer signal represents these resonances only poorly. Even though the signals show some similarities, the compensated AE transducer signal would not be enough to identify the resonances on its own. A possible explanation for the weak agreement, beside the calibration, can be the sensor itself. In comparison with the calibration plate, the sensor does change the system for the small discs by adding additional weight. The sensor rather is large $(\varnothing 19 \mathrm{~mm})$ and therefore violates the point assumption of the Green's function approach.

The absolute calibration seems more promising in boundary restricted systems if the exciting force and wave

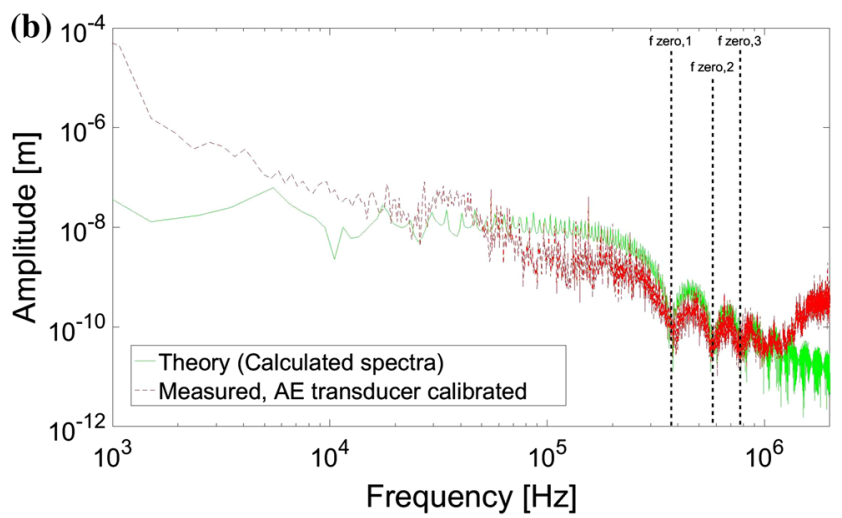



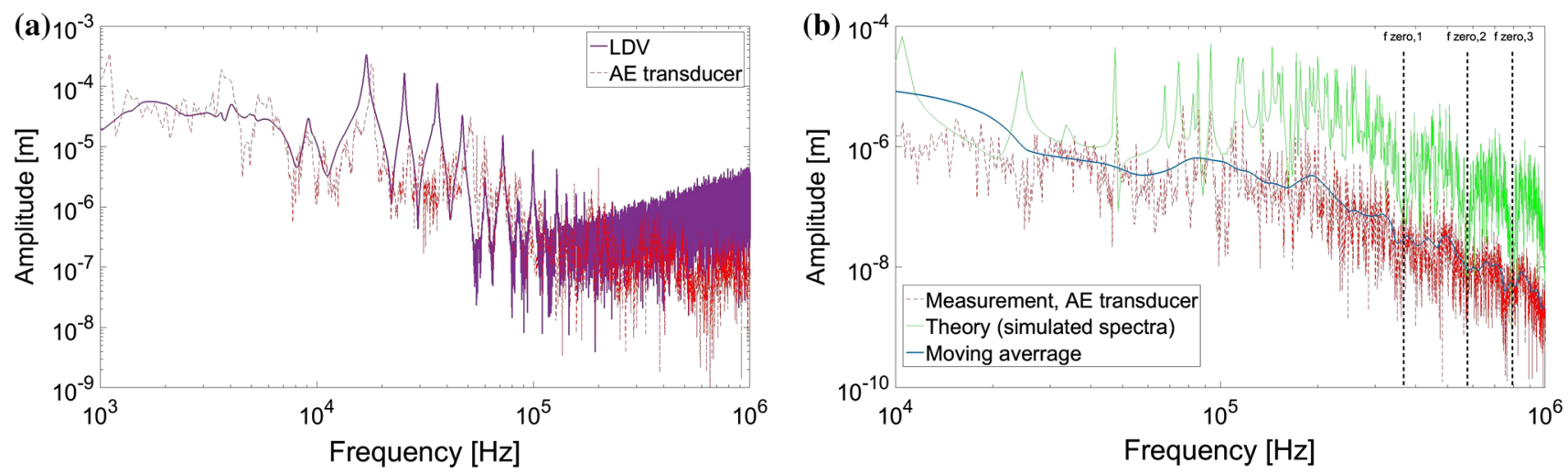

Fig. 8 Calibrated AE transducer measurements on boundary restricted systems. a Comparison of LDV measurement and AE transducer signal, b comparison of theoretical signal and $\mathrm{AE}$ transducer signal

reflections do not interact with each other (Fig. 8b). For measurements shown in Fig. 8b, a ball of the size of $\varnothing 1,5 \mathrm{~mm}$ was dropped onto a steel plate with a thickness of $29 \mathrm{~mm}$. By reducing the ball size and increasing the thickness, contact time was reduced $(5.1 \mu \mathrm{s})$ and reflection times were increased $\left(t_{\text {reflection } H}=9.831 \mu\right.$ s and $\left.t_{\text {reflection } R}=17.59 \mu \mathrm{s}\right)$. In this case, both excitation of the resonances and enhancing of zero frequencies was expected according to the calculated theoretical signal (green). Comparing the calculated signal and the compensated $\mathrm{AE}$ transducer signal (red), a good agreement of the zero frequencies can be observed. The dashed lines in Fig. $8 \mathrm{~b}$ are indicating the zero frequencies of first, second and third order. Local minimum of simulated and measured signals agree with these zero frequency positions. However, the excited resonances indicated by the theoretical signal are not clearly represented by the compensated $\mathrm{AE}$ transducer signal.

Sensor function compensated AE transducer signals is capable of identifying zero frequencies in boundary restricted systems. However, the method in combination with the used equipment is not able to identify the excited resonances, even though theory would suggest otherwise. Possible explanations could be as mentioned a violation of the point assumption. While the assumption is decent for the Hertzian contact, the used sensor head with a diameter of $19 \mathrm{~mm}$ violates this assumption. Another explanation could be nonlinearities in the sensor function. The calibration method assumes the sensor function $\left(I_{1}(\omega)\right)$ to be linear for a given frequency across the excitation amplitude, which might not be the case.

\subsection{Hertzian Contacts in Boundary Restricted Systems}

For boundary restricted systems, a finite element model was for this investigation developed to replace Hsus script which did not take any reflections into account. In combination with the Green's function approach, this model is also only valid for excitations which do not interact with the excited elastic wave. Comparing the calculated spectra (green solid line) in Fig. $8 \mathrm{~b}$ with the compensated $\mathrm{AE}$ transducer signal (red dashed line) shows that both are able to identify the zero frequencies. Zero frequencies according to McLaskey and Glaser [14] were calculated (11) and are indicated with the dashed vertical lines in both Figs. $8 \mathrm{~b}$ and 9. In Fig. $8 \mathrm{~b}$ both calculated and measured signals match the zero frequencies to a good degree. However, some deviation exists. This error gets more clearly in Fig. 9 where zero frequencies of the first three orders were extracted of all experiments and normalized by the contact time of the different impacts.

By normalization of the zero frequencies over the contact time $\left(t_{\mathrm{c}}\right)$, experiments with different ball sizes and drop heights are comparable. Figure 9 shows a visualization of normalized zero frequencies of several combinations of

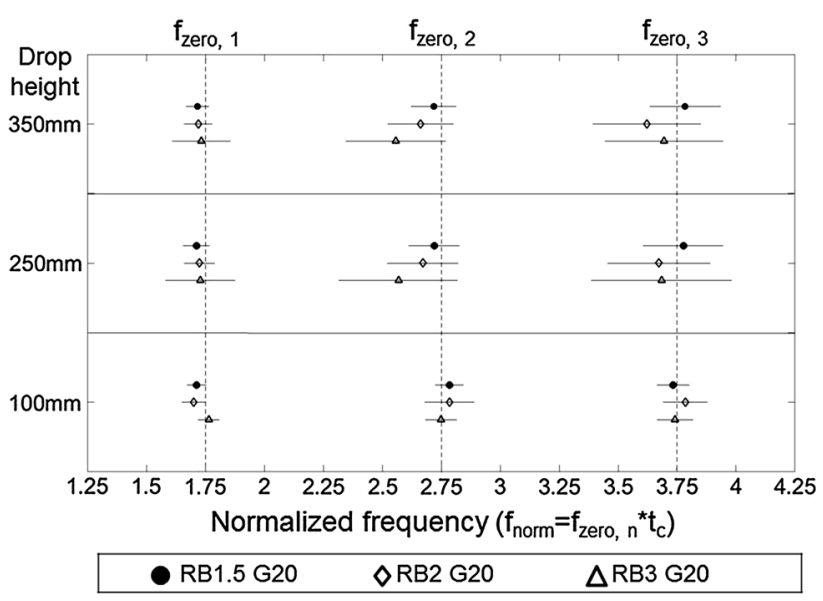

Fig. 9 Normalized frequencies of the first three zero frequencies for different balls and drop heights on a boundary restricted system (steel plate $29 \mathrm{~mm}$ thickness and $\varnothing 103.8 \mathrm{~mm}$ ) 
drop height and ball size. Each marker represents the mean value of eight measurements, and the error bars represent the standard deviation of the eight measurements. The results show that the identification of zero frequencies is clearly possible in boundary restricted systems. However, the failure margin increases in comparison with the case of an infinite plate [14].The failure margin shows a clear increasing trend with increased ball size. The reason for the increase is most probably the increase in contact time. For balls with a diameter of $3 \mathrm{~mm}$, the contact time $\left(t_{\mathrm{c}}\right)$ is almost as long as the reflection time $\left(t_{\text {reflection } H}\right)$. The contact time increases as well with a decrease in drop height. However, the results show a decreased failure margin. This is most likely caused by the increased spread of the impacts from increased heights as shown in Fig. 2.

By decreasing the reflection time $\left(t_{c}>t_{\text {reflection } H}\right)$ by decreasing plate thickness, zero frequencies could neither be detected by measurements nor by the FEM model based Green's functions approach. As the results indicate, elastic wave responses in a boundary restricted system turn into a pure resonance problem once the contact time exceeds the reflection times. As Fig. 10 shows, neither a change in drop height nor a change in ball size has an effect on the excited
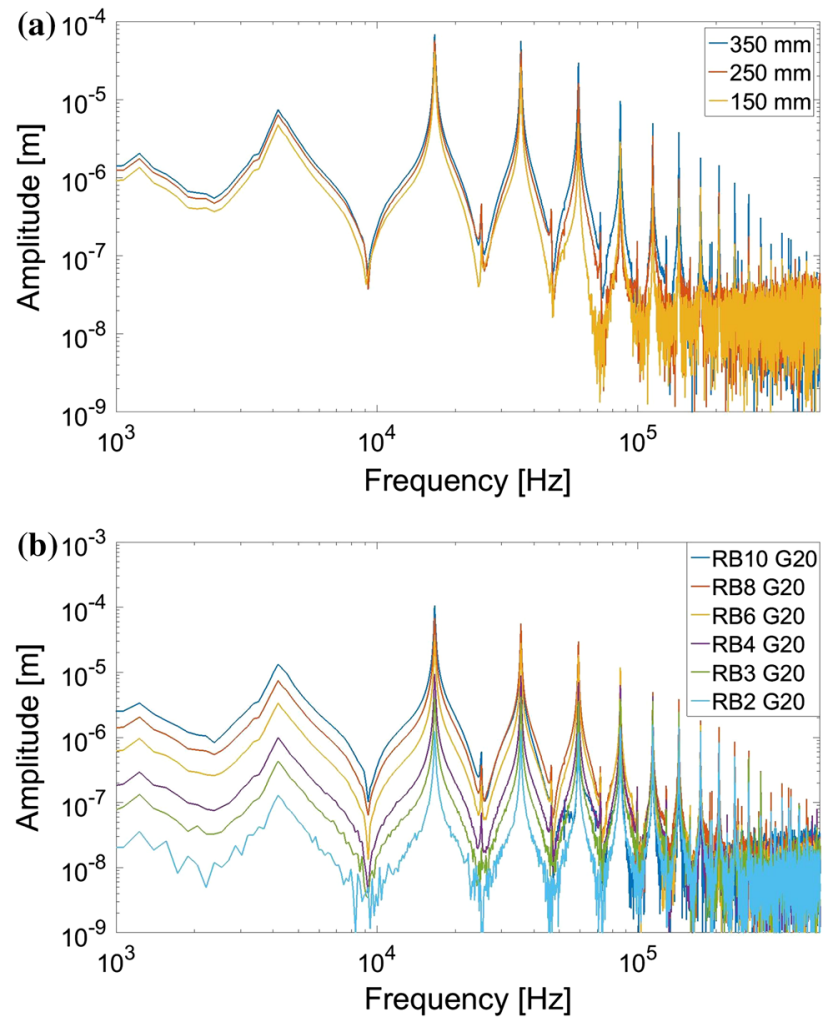

Fig. 10 Comparison of LDV measurements of different impact energies and contact times on a Steel 2511 plate of $5 \mathrm{~mm}$ thickness by changing drop height and ball size. a Drop height, $\mathbf{b}$ ball size

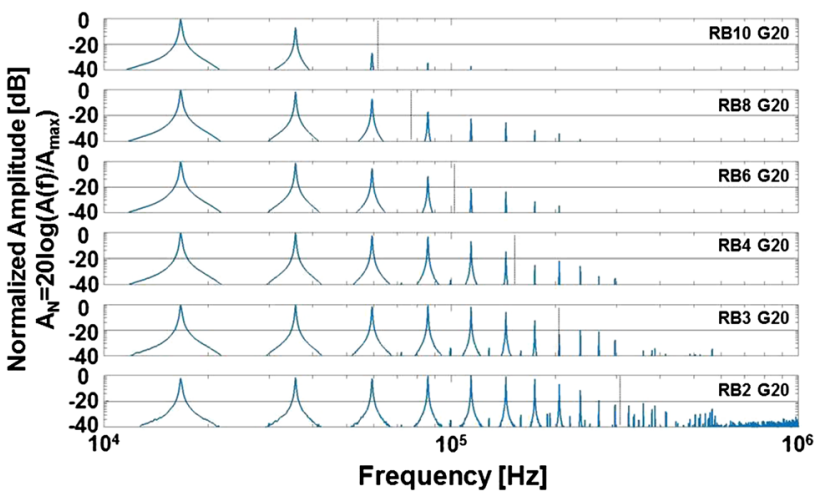

Fig. 11 Spectra of signals with normalized amplitude for different ball sizes

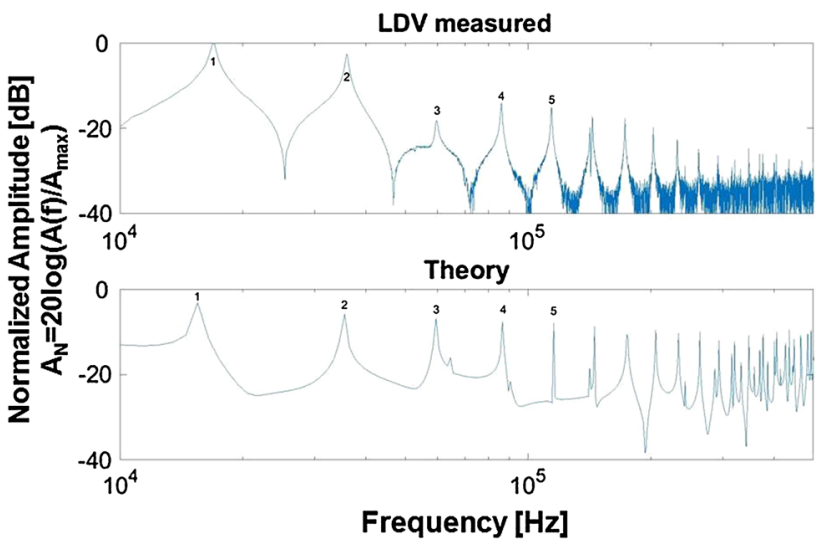

Fig. 12 Comparison of resonances calculated and measured

resonances. However, a change in contact time by changing ball size does change the cut-off as Fig. 11 indicates. Therefore, matches the first zero frequency $f_{\text {zero, } 1}$ (indicated as a dotted line in Fig. 11) to a good degree matches a $-20 \mathrm{~dB}$ cut-off.

Even though the Green's function approach is not valid for cases where initiating force and elastic wave interact, it still provides information about the location of the resonances. In Fig. 12 both the normalized spectra of an aluminium plate $5 \mathrm{~mm}$ obtained by calculation and by measurement are shown. The theoretical location and the measured location of the resonances are not identical, but show good agreement. Comparing calculated and measured resonances one to five (as indicated in Fig. 12) for different plates and ball sizes $(\varnothing 8 \mathrm{~mm}$ to $\varnothing 3 \mathrm{~mm}$ ) results in Fig. 13 . Except for resonance one, the agreement between calculated location and measured location is good considering the simplicity of the FEM model and the accuracy of the experiments. The larger deviation for the location of the first resonance can be partly explained by the larger 


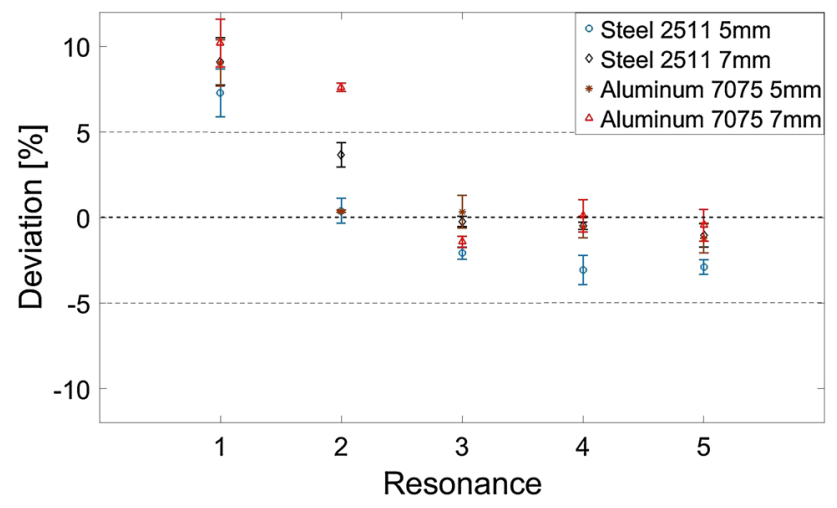

Fig. 13 Degree of agreement between resonances calculated and measured by the LDV

percentage difference between spectral lines at low frequencies and the short time obtained by calculation and by measurement of $1 \mathrm{~ms}$.

\section{Conclusions}

After evaluating calibration based on Green's functions, it can be concluded that the approach is also valid for boundary restricted systems with respect to zero frequencies. The calibration is, however, not accurate enough to detect resonances. This might be explained by violation of the assumptions of the Green's function approach by the sensor system or by a summation of measurement inaccuracy. The evaluation is, however, promising enough to investigate more accurate sensor systems which violate the assumptions of the Green's function approach to a lesser degree.

The investigation shows a clear difference between excitation times shorter than reflection times and excitation times longer than reflection times. In cases of independence of elastic waves and excitation force, the spectra contain both zero frequencies and resonances. Therefore, the zero frequencies follow Reed's [19] contact model, as suggested by McLaskey and Glaser [14]. However, if elastic wave and excitation force do interact, zero frequencies are not detectable anymore and the measured spectra exclusively contain resonances. Even though zero frequencies are not detectable in this case, the first zero frequency does affect the spectral signal by determining the $-20 \mathrm{~dB}$ cut-off frequency. Additionally, it was shown that the Green's function approach does provide partial information about the resonance location and is in line with measurements.

Acknowledgements The authors would like to acknowledge the financial support of SKF and VINNOVA, as well as the helpful discussions with researchers at SKF, among them Dr. Florin Tatar, SKF-ERC, Netherlands.

Open Access This article is distributed under the terms of the Creative Commons Attribution 4.0 International License (http://crea tivecommons.org/licenses/by/4.0/), which permits unrestricted use, distribution, and reproduction in any medium, provided you give appropriate credit to the original author(s) and the source, provide a link to the Creative Commons license, and indicate if changes were made.

\section{References}

1. Breckenridge, F., Tscheigg, C., Greenspan, M.: Acoustic emission: some applications of lamb's problem. J. Acoust. Soc. Am. 57, 626-631 (1975)

2. Bull, S.: Failure modes in scratch adhesion testing. Surf. Coat. Technol. 50(1), 25-32 (1991). doi:10.1016/0257-8972(91)901883. 18th International Conference on Metallurgical Coatings and Thin Films, San Diego, APR 22-26 (1991)

3. Derakhshan, O., Hougthon, J., Jones, R., March, P.: Cavitation monitoring of hydroturbines with RMS acoutic emission. In: Acoustic Emission: Current Practice and Future Directions, American Society for Testing and Materials Special Technical Publication, vol. 1077, pp. 305-315. American Society for Nondestructive testing, IEEE (1991). doi:10.1520/STP19102S. Symposium on World Meeting on Acoustic Emission, Charlotte, MAR 20-23 (1989)

4. Dolinsek, S., Kopac, J.: Acoustic emission signals for tool wear identification. WEAR 225(1), 295-303 (1999). doi:10.1016/ S0043-1648(98)00363-9. 12th International Conference on Wear of Materials, ATLANTA, APR 25-29 (1999)

5. Glaser, S., Weiss, G., Johnson, L.: Body waves recorded inside an elastic half-space by an embedded, wideband velocity sensor. J. Acoust. Soc. Am. 104(3, 1), 1404-1412 (1998). doi:10.1121/1. 424350

6. Gross, S., Fineberg, J., Marder, M., McCormick, W., Swinney, H.: Acoustic emission from rapidly moving cracks. Phys. Rev. Lett. 71(19), 3162-3165 (1993). doi:10.1103/PhysRevLett.71. 3162

7. Hamel, M., Addali, A., Mba, D.: Monitoring oil film regimes with acoustic emission. Proc. Inst. Mech. Eng. Part J: J. Eng. Tribol. 228(2), 223-231 (2014). doi:10.1177/1350650113503631

8. Han, Z., Luo, H., Wang, H.: Effects of strain rate and notch on acoustic emission during the tensile deformation of a discontinuous yielding material. Mater. Sci. Eng. A Struct. Mater. Prop. Microstruct. Process. 528(13-14), 4372-4380 (2011). doi:10. 1016/j.msea.2011.02.042

9. Hsu, N.: Dynamic greens functions of an infinite plate-a computer program. Technical Report No. NBSIR 85-3234, National Bureau of Standards, Center of Manufacturing Engineering, Gaithersburg (1985)

10. Jacobs, L., Woolsey, C.: Transfer functions for acoutic-emission transducers using Laser interferometry. J. Acoust. Soc. Am. 94(6), 3506-3508 (1993). doi:10.1121/1.407205

11. Johnson, P.A., Savage, H., Knuth, M., Gomberg, J., Marone, C.: Effects of acoustic waves on stick-slip in granular media and implications for earthquakes. Nature 451(7174), 57-U5 (2008). doi:10.1038/nature06440

12. Kundu, T., Das, S., Jata, K.V.: Point of impact prediction in isotropic and anisotropic plates from the acoustic emission data. J. Acoust. Soc. Am. 122(4), 2057-2066 (2007). doi:10.1121/1. 2775322 
13. Liu, S., Datta, S.: Scattering of ultrasonic wave by cracks in a plate. J. Appl. Mech. Trans. ASME 60(2), 352-357 (1993). doi:10.1115/1.2900800. 1st Joint Meeting of the American Society of Civil Engineers and the American Society of Mechanical Engineers, SES, Charlottesville, Jun 06-09 (1993)

14. McLaskey, G.C., Glaser, S.D.: Hertzian impact: experimental study of the force pulse and resulting stress waves. J. Acoust. Soc. Am. 128(3), 1087-1096 (2010). doi:10.1121/1.3466847

15. McLaskey, G.C., Glaser, S.D.: Acoustic emission sensor calibration for absolute source measurements. J. Nondestruct. Eval. 31(2), 157-168 (2012). doi:10.1007/s10921-012-0131-2

16. Miettinen, J., Andersson, P.: Acoustic emission of rolling bearings lubricated with contaminated grease. Tribol. Int. 33(11), 777-787 (2000)

17. Miettinen, J., Pataniitty, P.: Operation monitoring of grease lubricated rolling bearings by acoustic emission measurements. Int. J. COMADEM 7(2), 2-11 (2004)
18. Niknam, S.A., Songmene, V., Au, Y.H.J.: The use of acoustic emission information to distinguish between dry and lubricated rolling element bearings in low-speed rotating machines. Int J. Adv. Manuf. Technol. 69(9-12), 2679-2689 (2013). doi:10. 1007/s00170-013-5222-4

19. Reed, J.: Energy losses due to elastic wave propagation during an elastic impact. J. Phys. D Appl. Phys. 18, 2329-2337 (1985)

20. Schnabel, S., Marklund, P., Larsson, R.: Study of the short-term effect of $\mathrm{Fe}_{3} \mathrm{O}_{4}$ particles in rolling element bearings: Observation of vibration, friction and change of surface topography of contaminated angular contact ball bearings. Proc. Inst. Mech. Eng. Part J: J. Eng. Tribol. 228(10, SI), 1063-1070 (2014). doi:10.1177/1350650114526582

21. Tandon, N., Ramakrishna, K., Yadava, G.: Condition monitoring of electric motor ball bearings for the detection of grease contaminants. Tribol. Int. 40(1), 29-36 (2007) 